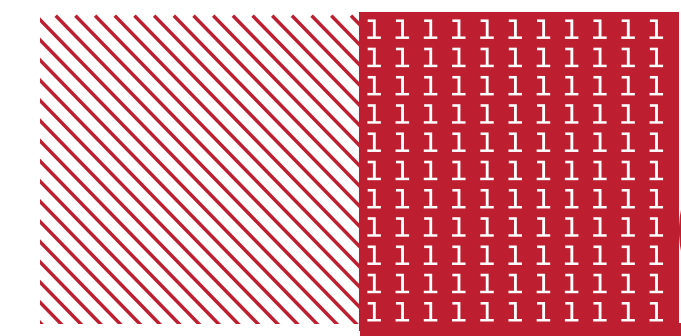

\title{
AMBIENTES DE APRENDIZAJE EN CONTEXTO: ESTUDIO DE CASO EN CURSOS UNIVERSITARIOS DE MATEMÁTICAS PREVIAS AL CALCULO
}

Learning Environments in Context: Case Study in University Courses of Mathematics Prior to Calculus

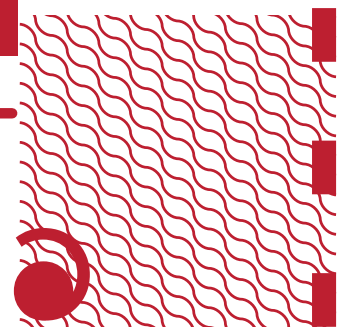

Ambientes de aprendizagem em contexto: Estudo de caso em cursos universitários de Matemática prévias ao cálculo

Henry Arley Taquez Quenguan'

Departamento de Pedagogía,

Universidad Icesi, Cali, Colombia.

hataquez@icesi.edu.co

(D): $\underline{0000-0003-3298-9979}$
Hendel Yaker Agudelo ${ }^{2}$

Departamento de matemáticas y estadística, Universidad Icesi, Cali, Colombia. hyaker@icesi.edu.co

(D): $0000-0002-8766-4410$ 


\author{
Ángela María Bedoya Urrego ${ }^{3}$ \\ Departamento de gestión \\ organizacional, Universidad \\ Icesi, Cali, Colombia. \\ angela.bedoya@u.icesi.edu.co \\ (D): $0000-0002-5027-2560$
}

\author{
Alexander Parra ${ }^{4}$ \\ Institución educativa José Antonio \\ Galán, Yumbo, Valle. \\ profealexparra@gmail.com \\ (D): $\underline{0000-0002-3258-4165}$
}

\section{Recepción: 15/11/2020 • Aprobación: 30/03/2021}

Resumen: A nivel universitario se ha identificado un problema de bajo rendimiento en los cursos de matemática de formación básica para Ciencias e Ingeniería. En la Universidad Icesi se inició un proyecto de investigación con la caracterización del ambiente de aprendizaje en tres cursos de Álgebra y Funciones. Se formularon categorías de análisis para cuatro dimensiones constitutivas de un ambiente de aprendizaje y se hicieron observaciones centradas en el tema de funciones. En el momento de inicio del proyecto los cursos son presenciales. Las conclusiones sobre las categorías de análisis planteadas señalan la importancia de las condiciones físicas del espacio de interacción (aula de clase) y el efecto negativo, sobre la reflexividad del profesor, que tiene la premura por cubrir un programa estructurado por contenidos. En el artículo se plantean algunos referentes teóricos desde los cuales se pueden proponer soluciones viables al problema de bajo rendimiento, atendiendo a las características contextualizadas de los ambientes de aprendizaje. La socialización del resultado del estudio se constituyó en uno de los factores que impulsó la actual reforma del curso de Álgebra y Funciones, cuyo desarrollo y seguimiento ha sido impactado por la necesidad de migrar a ambientes virtuales durante la pandemia en el año 2020.

Palabras claves: Ambiente de aprendizaje; Álgebra y funciones; GeoGebra; Bajo rendimiento.

Resumo: A nível universitário tem se identificado um problema de baixo rendimento entre os cursos de matemática de formação básica para ciências e engenharia. $\mathrm{Na}$ universidade Icesi se iniciou um projeto de pesquisa com a caracterização do ambiente de aprendizagem em três cursos de álgebra e funções. Formularam-se categorias de analise para quatro dimensões constitutivas de um ambiente de aprendizagem e foi feito observações centradas no tema de funções. 
No momento do início do projeto os cursos são presenciais. As conclusões sobre as categorias de análise propostas assinalam a importância das condições físicas do espaço de interação (sala de aula) e o efeito negativo, sobre a flexibilidade do professor, que tem a pressa por cobrir um programa estruturado por conteúdo. No artigo se propõe alguns referentes teóricos desde os quais podem-se propor soluções viáveis ao problema de baixo rendimento, atendendo as características contextualizadas dos ambientes de aprendizagem. A socialização do resultado do estudo construiu-se em um dos fatores que impulsou a atual reforma do curso de Álgebra e Funções, cujo desenvolvimento e seguimento tem sido impactado pela necessidade de migra a ambientes virtuais durante a pandemia em 2020.

Palavras-chave: Ambiente de aprendizagem; Álgebra e funções; GeoGebra; Baixo desempenho.

Abstract: At a university level, it has been identified a problem of low performance within the courses of basic mathematic instruction in the Sciences and Engineering programmes. At the Icesi University, a research project has begun with the characterization of the learning environment within three courses of Algebra and Functions. Analysis categories were formulated for four constitutive dimensions of a learning environment and some observations were made focused in the subject of functions. At the beginning of the project, the courses are face-to-face courses. The stated conclusions about analysis categories remark the importance of appropriate conditions of the interaction spaces (i.e. classrooms), and the negative effect these conditions can have on the teachers reflections, who have the pressure of time for covering a structural content program. In this article, are raised some theoretical referents from which viable solutions for the low performance problem can be suggested; all of this, taking into account the particularly contextualized characteristics of the learning environments. The dissemination of the research result has become one of the main factors to promote the present re-design of the course of Algebra and Functions. The development and following of this process has been affected by the necessity of migration towards virtual environments during the Covid-19 pandemic since the year 2020.

Key words: Learning environments; Algebra and Functions; GeoGebra; Low performance.

Procedencia: Este artículo no recibió financiación. 


\section{¿Cómo citar este artículo? / How to quote this article?}

Taquez Quenguan, H., Yaker Agudelo, H., Bedoya Urrego, A. M., y Parra, A. (2020). Ambientes de aprendizaje en contexto: estudio de caso en cursos universitarios de matemáticas previas al cálculo. Praxis, Educación y Pedagogía (5), 6-27. https://doi.org/10.25100/praxis_educacion.v0i5.10779

\section{Introducción}

Las grandes deficiencias en conocimientos matemáticos fundamentales y los malos desempeños en ejecuciones procedimentales a nivel de aritmética y álgebra básica de los estudiantes que inician estudios universitarios de Ciencias e Ingeniería, son motivo de constante preocupación para

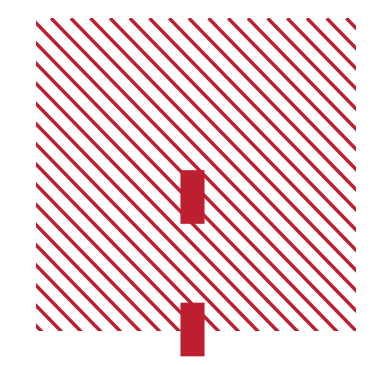
los profesores de matemáticas y para los estamentos directivos de estas carreras en varias de las principales universidades de la ciudad de Cali. Resulta tanto o más alarmante el bajo impacto que tiene el primer curso de matemáticas universitarias en la transformación de estas conductas de entrada, a pesar de todo el proceso llevado a cabo durante el semestre.

En la fase inicial de este proyecto nos propusimos caracterizar el ambiente de aprendizaje en cursos de Álgebra y Funciones (AyF) siguiendo las pautas del Ministerio de Educación Nacional (MEN) en su documento orientador del foro educativo de 2014 (MEN, 2014). En nuestro estudio hicimos la exploración en tres aulas distintas, con sus respectivos profesores y grupos de estudiantes.

La escogencia de ambiente de aprendizaje como foco de análisis en la fase inicial del proyecto, y del concepto de función como objeto de observación, están naturalmente asociadas a los referentes teóricos que enmarcan el trabajo de los investigadores, de donde se extraen algunos elementos fundamentales para abordar el problema: i) la necesidad de considerar los contextos institucionales en la interpretación de los fenómenos de aprendizaje y en las propuestas didácticas; ii) la concepción del aprendizaje como un proceso constructivo y cooperativo, que se produce en un contexto particular; iii) la necesidad de darle sentido a la matemática escolar como actividad matemática y iv) la comprensión de la dimensión semiótica de la actividad matemática, donde cobra especial importancia el concepto de función, en su doble naturaleza de objeto y de proceso, 
que involucra las complejas nociones de variable y covariación, y se convierte en elemento central del discurso matemático escolar. Una ventaja importante del enfoque cualitativo utilizado es que nos da la posibilidad de plantear preguntas, incluso algunos supuestos, en cualquier momento del proceso, antes o después de la recolección y el análisis de datos. En este sentido, aceptamos como premisa básica que las universidades ofrecen un primer curso de matemáticas previas al cálculo, porque han establecido que los estudiantes que inician su educación superior necesitan una revisión y refuerzo de su matemática de bachillerato. En este estudio nos centramos en entender las posibles relaciones entre los ambientes de aprendizaje y la poca incidencia en la formación de los estudiantes que tienen los primeros cursos de matemáticas universitarias.

\section{Marco Teórico}

Algunos investigadores en educación matemática han abordado el proceso de transición de la educación media a la superior y han estudiado las concepciones predominantes acerca de la matemática en los profesores universitarios, junto con las prácticas docentes asociadas (Artigue, 2016, 2017; Bosch, Fonseca y Gascón, 2004; Gueudet, Bosch, DiSessa, Kwon, \& Verschaffe, 2016; Rach \& Heinze, 2017; Rodríguez-Cisneros y Perdomo-Díaz, 2019; Tall, 1992). Se estima que la mayoría de los profesores no creen que la investigación en educación ofrezca resultados prácticos para el trabajo en el aula y tienden a orientar su labor docente influenciados por la filosofía en la que fueron formados, lo que normalmente conduce al modelo pedagógico tradicional basado en la transmisión del conocimiento, la memoria y la repetición.

En MEN (2014) se plantean cuatro dimensiones para la construcción de un ambiente de aprendizaje, que se suponen inmersas en contextos matemáticos y contextos sociales específicos: i) El espacio físico donde se realizan las actividades e interacciones; ii) las actividades de aprendizaje; iii) los recursos que tienen una intención didáctica y iv) el conjunto de interacciones posibles entre todos los componentes y actores del ambiente. Adoptando esta propuesta como base, el equipo construyó las categorías de análisis que sirvieron de guía para la caracterización de los ambientes planteados en el estudio exploratorio. Para esta construcción se usaron como soporte algunas ideas fundamentales tomadas de la literatura revisada.

En cuanto a la dimensión semiótica de la actividad matemática, es necesario atender los conflictos y posibilidades que se generan de la conjugación de factores ineludibles como: $i$ ) las actividades e interacciones de los sujetos con los objetos de conocimiento, necesariamente deben ser elaboradas por los sujetos en un proceso de simbolización; ii) los objetos matemáticos no son accesibles 
directamente a los sentidos, pues su sustancia es simbólica y, por tanto, la actividad básica del estudiante es utilizar diversos niveles de representación para la construcción del sentido; iii) la transformación del uso de tecnología en la educación matemática está generando nuevas formas de representación que no son neutrales epistemológicamente y que estamos apenas empezando a vislumbrar (D'amore,

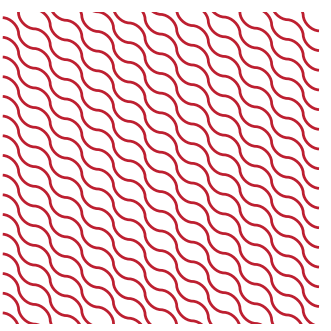
2004; Hegedus \& Moreno-Armella, 2011).

El concepto de función es fundamental en el discurso matemático escolar, principalmente por su rol en las representaciones, en diversos registros, de los modelos matemáticos. La literatura reporta varios estudios relacionados con la complejidad en la enseñanza y el aprendizaje del tema de funciones (Dominguez \& Cerqueira, 2021; Hitt \& González-Martín, 2016; Nyikahadzoyi, 2015; Sierpinska, 1992).

En las últimas dos décadas se ha abordado en profundidad el tema de la mediación tecnológica en la educación matemática (Artigue, 2011; Drijvers et al., 2009; Moreno-Armella \& Brady, 2018; Santos-Trigo, 2019; Santos-Trigo, Aguilar-Magallón \& Reyes-Martínez, 2019; Santos-Trigo \& Moreno-Armella, 2016; Santos Trigo y Benítez Mojica, 2003; Tall, 2000); se acepta que las ideas y conceptos abstractos de las matemáticas se convierten en realidades cuando se pueden manipular y transformar, lo cual es posible con el uso de las herramientas computacionales; se aborda el tema del impacto de las herramientas tecnológicas en los procesos de representación de los objetos matemáticos, planteando que las tecnologías influyen tanto en la forma de enseñar como en la manera de aprender. Se resalta, en general, la importancia del empleo de diferentes formas de representación de los conceptos matemáticos y se critica la tendencia tradicional de privilegiar las representaciones algebraicas.

\section{Metodología}

Para la investigación se utilizó un estudio de caso, privilegiando una metodología cualitativa donde se pretende comprender, profundizar e interpretar el fenómeno de estudio.

\section{El Estudio de Caso: Recolección de la información}

En este estudio, se hace referencia a tres profesores: P1, P2 y P3. Cada profesor con su grupo de estudiantes de AyF elegido para observación constituye un caso. Los estudiantes son jóvenes entre los 16 y 18 años. La recolección de la información se realizó durante las 6 semanas de clase dedicadas al tema de 
funciones. El investigador principal, con la colaboración de dos monitoras, hicieron la observación directa de las clases de cada uno de los profesores; al final del proceso de observación se realizaron entrevistas semiestructuradas para cada profesor y se revisaron los cuadernos de un estudiante por cada curso. La observación fue guiada con el instrumento construido de las categorías de análisis (cuya estructura se muestra en los resultados) y se realizó de manera abierta, es decir, el observador anotaba en su bitácora el desarrollo de la clase sin seguir ninguna estructura previa, pues se quería observar el ambiente de aprendizaje en su modo original y natural. La información resultante de la observación y las entrevistas se digitó en el software para investigación cualitativa Atlas.ti, donde se codificó de acuerdo con las categorías de análisis. Finalmente se hizo un análisis utilizando triangulación, para identificar con un alto grado de profundidad las relaciones que se establecen entre las diferentes fuentes de información utilizadas.

\section{Resultados}

A continuación, mostramos en detalle los resultados obtenidos por cada categoría del ambiente de aprendizaje.

\section{Entorno}

a. Componentes físicos. Salones con capacidad para 25 estudiantes, dotados con sillas individuales, aire acondicionado, computador para el profesor y video beam. Un tablero de borrado en seco con un borrador, el profesor carga los marcadores. Los tres profesores entrevistados (P1, P2, P3) coincidieron en que la capacidad de los salones en los que se desarrolla la clase de álgebra y funciones es muy poca para la cantidad de estudiantes que toman este curso.

b. Organización. Generalmente se inicia con una organización tradicional, donde la comunicación fluye del profesor al alumno. Hay un momento de la clase donde se reacomodan los puestos para formar grupos de trabajo. Durante actividades o talleres en clase, se fomenta una organización más activa, donde los estudiantes se sitúan por toda el aula, de acuerdo a sus gustos para formar grupos de trabajo y desarrollar la actividad.

\section{Actividades de aprendizaje}

a. Actividades correspondientes al rol del profesor. El programa plantea una estructura de clase en tres momentos: i) revisión de la agenda del día y de la ruta de clase (guía de lecturas y actividades que los 
estudiantes deben llevar preparadas a la clase), que están disponibles en Moodle. Este momento debe tomar aproximadamente media hora. ii) Se proponen ejercicios para la clase, normalmente tomados del texto guía y, generalmente, formando grupos de trabajo entre los estudiantes. Este momento debe tomar aproximadamente una hora. Los objetivos de aprendizaje están determinados por el texto guía y la selección particular que se hizo en el programa, que el profesor sigue prácticamente al pie de la letra. iii) Se presenta la agenda para la próxima clase, con explicaciones e instrucciones del profesor. Este momento debe tomar aproximadamente media hora. De acuerdo con las observaciones, las actividades que realizan los tres profesores se pueden clasificar en cuatro tipos: complemento del libro, reproducción del libro, resolución de inquietudes y procedimientos paso a paso.

\section{b. Actividades correspondientes al rol del estudiante.}

i. Individuales. En sus cuadernos desarrollan, previo a la clase, las actividades propuestas en la ruta. Unos pocos plantean interrogantes concretos en el primer momento de clase (la mayoría se limita a hacer coro a expresiones del tipo "estaba difícil" o "no entendî").

ii. Grupales. Atendiendo indicaciones del profesor, se conforman grupos de entre 4 o 5 estudiantes, que reacomodan sus puestos individuales para organizar especies de "mesas redondas". Generalmente se comparten sus respuestas del trabajo previo y se forman pequeñas discusiones en las que, normalmente, tiene que mediar el profesor.

c. Evaluación. Los tres profesores afirmaron en las entrevistas que la evaluación formativa hace parte esencial de su trabajo, ya que todo el tiempo hacen observación y seguimiento en los talleres en clase y revisión de rutas de clase. Los profesores aprovechan el momento en que los estudiantes están trabajando en grupo, para acercarse a ellos y ver cómo van en su proceso de aprendizaje, hacer y contestar preguntas que les permitan saber cómo va el estudiante y qué tanto está entendiendo el tema que se está trabajando. Los tres afirman que con base en esa evaluación formativa y la que realizan en la revisión de quices, pruebas cortas y parciales, identifican qué temas tienen que seguir trabajando y cuáles están claros para los estudiantes. Además, también les ayuda a ubicar aquellos estudiantes que necesitan un acompañamiento

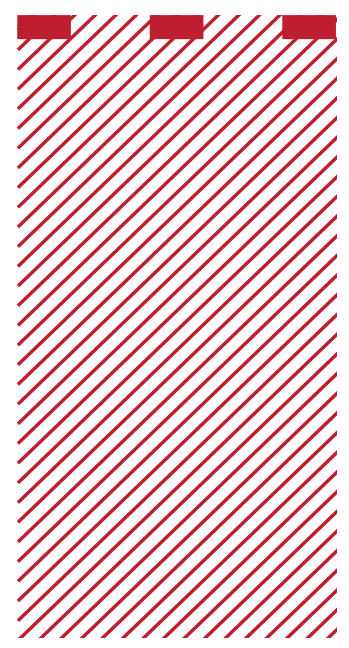




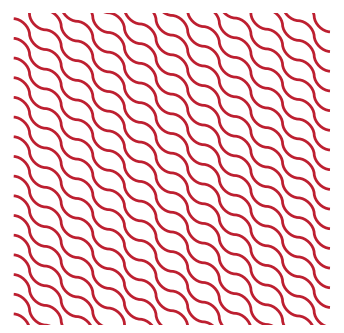

más personalizado para acercarse más a ellos en clase y sugerirles que acudan a los servicios de tutorías que les ofrece la universidad. La evaluación final, es creada conjuntamente por todos los profesores del curso y se busca evaluar los objetivos generales y terminales del mismo. Los tres profesores resaltaron que algunas herramientas como Moodle, Kahoot y Khan academy resultan ser de gran ayuda para realizar quices, ya que se califican automáticamente y ofrecen algunas estadísticas. De lo observado cabe resaltar, siguiendo la estructura del instrumento:

i. Mecanismos. Se revisa el trabajo en la ruta de clase a cuatro o cinco estudiantes por clase. Algunas veces se pide entregar un informe del trabajo en clase por grupo. Hay simulacros en la plataforma, que se deben trabajar en casa, o en salas de cómputo de la universidad. Se realizan pruebas cortas, exámenes parciales y un final.

ii. Instrumentos. Planillas que contienen los nombres y varias columnas. Por lo general allí se registra el resultado de la revisión del trabajo previo de clase y el trabajo de clase, con indicadores del tipo cumplió o no cumplió o calificaciones en una escala de 1 a 5. Pruebas escritas con preguntas tomadas del texto guía y de los talleres trabajados en clase. En general, tales pruebas tienen en promedio diez preguntas y se califican en una escala de 1 a 5 , con diferentes ponderaciones en los puntos, pero sin una rúbrica particular. Los simulacros utilizan las posibilidades tecnológicas de la plataforma Moodle y consisten en general de pruebas que combinan preguntas abiertas y preguntas de selección múltiple. El esquema de evaluación sumativa es: preparación y trabajo en clase $10 \%$, dos pruebas cortas $20 \%$, dos parciales $40 \%$ y un examen final $30 \%$.

iii. Momentos. La programación de pruebas escritas se explicita en el programa: la preparación y trabajo en clase se evalúa en forma permanente, las pruebas cortas se aplican en las semanas 5 y 10 , los parciales en las semanas 7 y 13 y el examen final en la semana 18, dos semanas después de terminadas las clases. Los simulacros se aplican los fines de semana previos a prueba escrita y se hace discusión de los resultados (explicaciones del profesor) en la primera clase de la semana de la prueba respectiva. Las pruebas escritas se retornan calificadas y, por lo general, el día de la entrega el profesor hace la explicación de los puntos en los que detecta mayor confusión por parte de los estudiantes. 


\section{Recursos para el aprendizaje}

a. Programa oficial. El contenido del programa es tomado fundamentalmente del texto guía, pero hay una unidad cero o de nivelación para la cual se utilizan notas de clase de profesores de la universidad. Los objetivos generales y terminales se determinan a partir de un ejercicio de reflexión sobre los temas seleccionados del texto guía. Las rutas de clase son guías para las actividades diarias, que incluyen el trabajo previo a la clase que debe hacer el estudiante. Las rutas son construidas por el equipo de profesores que se coordinan para orientar el curso en los diferentes grupos. El elemento central en las rutas es la selección cuidadosa de los ejercicios del texto que pondrán en juego las ideas principales de los contenidos programáticos, con un tratamiento progresivo en nivel de dificultad y de posible aplicabilidad de los conceptos.

i. Alineación curricular. Las actividades de clase se orientan más a cumplir con el cubrimiento de los contenidos, tal como los presenta el texto guía y la ruta de clase. Las pruebas escritas, en su mayoría, se someten a un filtro en la coordinación del curso, donde básicamente se contrasta cada pregunta contra el listado de objetivos específicos del programa, para decidir su pertinencia y nivel de dificultad.

ii. Reflexividad del profesor. Los profesores siguen las rutas de clase. Normalmente toman la decisión de hacer presentaciones magistrales cuando sienten la necesidad de avanzar en el cubrimiento de los contenidos. No es frecuente que se tomen decisiones de cambios o reorientación de las actividades de clase, basadas en un análisis de las inquietudes o dificultades manifestadas por los estudiantes. El único momento que convoca a una reflexión sobre lo que está pasando en el curso, desde la perspectiva de los resultados, es el que sigue a la calificación de las pruebas escritas. La discusión colegiada suele centrarse en el nivel de dificultad que tuvieron las preguntas, aunque normalmente surge como factor, declarado por los profesores como agravante, la falta de compromiso de los estudiantes.

b. Apoyos tecnológicos. Se utiliza la plataforma Moodle para disponer la información del curso (programa, rutas de clase, copias del texto, talleres, videos) y para hacer exámenes (simulacros) como preparación a las evaluaciones programadas. En contadas ocasiones se propone a los estudiantes usar sus equipos (computadores

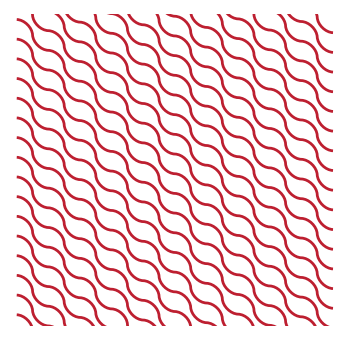


portátiles o teléfonos) para hacer alguna indagación en internet. También en contadas ocasiones se utiliza software de matemáticas (principalmente GeoGebra), para ilustrar el comportamiento de algunas funciones mediante su gráfica, o para efectuar algunos cálculos algebraicos de cierta complejidad. La herramienta principal es el tablero fijo que hay en el salón, donde se ponen en juego las explicaciones del profesor y de los estudiantes.

c. Discurso matemático.

i. Enfoque del concepto de función que adopta el profesor. Se sigue el enfoque esencialmente conjuntista del texto guía: función como un tipo particular de relación (conjunto de parejas ordenadas). La idea de covariación entre cantidades de magnitud se pone en juego solo con algunos de los problemas que se proponen en el texto. No se realizan actividades experimentales o que impliquen recolección de datos y análisis de información a partir de situaciones cotidianas.

ii. Registros de representación. Se utiliza el registro algebraico para construir tablas (con pocos datos), y a partir de las tablas se pasa al registro gráfico. Las limitaciones técnicas para llenar las tablas y para dibujar en el tablero, dejan el peso del análisis en la representación algebraica: la fórmula. Aquí la atención suele centrarse más en la parte operativa con los símbolos (cómo simplificar, cómo evaluar). Los estudiantes evidencian preferencia por la construcción de tablas, que luego traducen en puntos del plano cartesiano para producir finalmente, por un proceso implícito de interpolación, las gráficas de las funciones. En las observaciones y en la revisión de los cuadernos quedó claro que los tres profesores pocas veces privilegian el registro de representación verbal en clase. Por ende, cuando el profesor reclama comunicación de ideas en el lenguaje natural, los estudiantes presentan inconvenientes para hacerlo, les cuesta interpretar la información que se les suministra y la que sale de los resultados algebraicos que consiguen. En las entrevistas, todos los profesores coincidieron en que, aunque desde

-

..................

(.................

(..................

(..................

(................

(n...............

(..................

(................. la época escolar, la parte algebraica es la que más se trabaja con los estudiantes, resulta ser que tienen muchas dificultades en esto porque se han aprendido los procesos de una forma muy mecánica y memorística. En general, los estudiantes muestran interés por ver las representaciones gráficas de lo que han 
hecho algebraicamente, pero a pesar de esto, tienen dificultades a la hora de pasar de otro registro, al registro gráfico.

iii. Procesos de actividad matemática. Se privilegian los procesos de ejecutar procedimientos y algoritmos. Se resuelven problemas del texto y, alrededor de ellos, se promueven procesos de razonamiento, normalmente guiados por sugerencias y explicaciones del profesor. En general se hacen algunos llamados de atención sobre los problemas de comunicación que evidencian los estudiantes cuando escriben o intentan verbalizar sus respuestas, pero no hay un seguimiento sistemático ni un tratamiento específico de estos problemas. Los profesores utilizan primordialmente un lenguaje técnico al dirigirse a los estudiantes y, con bastante frecuencia, cierran discusiones con monólogos explicativos apresurados, por la prisa de cumplir con los tiempos de clase. En las observaciones se evidenció que la mayoría de estudiantes utilizan predominantemente algoritmos algebraicos. Cuando se les plantean problemas que exigen un proceso de razonamiento que trascienda los símbolos algebraicos, muchos estudiantes abandonan el intento, o encuentran muchas dificultades en su resolución.

\section{Interacciones}

a. Estudiante - entorno y profesor - entorno. La disposición de los puestos y de los útiles de los estudiantes dificulta la movilidad en el salón. En general los salones están bien climatizados, pero hay fallas frecuentes de conectividad y el computador del profesor y el video beam suelen presentar problemas.

b. Estudiante - estudiante. Los estudiantes se ubican en el salón rodeándose de sus amigos y compañeros de confianza. Así son frecuentes algunas charlas personales que distraen la atención de la clase, pero también comparten entre ellos sus dudas, opiniones y respuestas. Algunos profesores proponen actividades grupales imponiendo la integración de estudiantes con intenciones específicas: por ejemplo, que un estudiante con dificultades en algún tema pueda compartir con compañeros que tienen mejor comprensión del mismo. Es más común que los estudiantes escojan, por afinidades, sus grupos de trabajo. Estos grupos suelen tener 4 o 5 integrantes, un líder que destaca y uno o dos estudiantes que aportan muy poco o nada en la actividad.

c. Estudiante - profesor. En general es más frecuente la comunicación unidireccional del profesor al estudiante, mientras se dan 


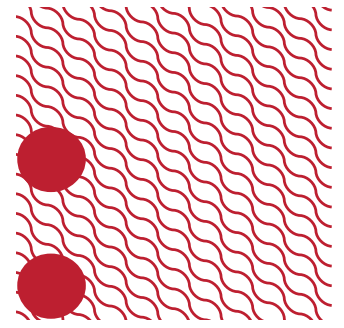

explicaciones o instrucciones. En tales circunstancias, también es frecuente que el profesor deba interrumpir su discurso para llamar la atención de estudiantes distraídos. Cuando hay actividades grupales es común que el profesor se acerque a cada grupo y se integre unos minutos en un intercambio de preguntas y respuestas. Durante las actividades individuales o grupales propuestas en la clase, algunos estudiantes se dirigen al puesto del profesor para pedir de forma particular explicaciones o sugerencias. Lo normal es que todas estas interacciones sean formales y relacionadas con la materia de estudio. En los tres grupos observados, el factor común fue que los estudiantes hicieron muchas más preguntas al profesor cuando estaban trabajando de forma grupal, ya que éste se acercaba a cada grupo y respondía las dudas de forma más personalizada. Cuando el profesor está al frente, dirigiéndose a todos, los estudiantes hacen pocas preguntas. En ambos casos, los estudiantes buscan sobre todo la repetición de algún procedimiento o explicación paso a paso.

\section{Discusión}

Planteamos a continuación las ideas que nos parecen más relevantes al analizar los resultados a la luz del marco teórico que estructura las categorías de análisis.

\section{Entorno}

Las observaciones muestran que hay una mejor dinámica en el aula cuando se promueve la conformación de grupos de trabajo. Esto implica requerimientos en varias dimensiones; la institución educativa debe pensar en la infraestructura de sus espacios físicos y en la dotación adecuada de las aulas de clase; el profesor debe considerar en su planeación las propuestas de actividades y los recursos de apoyo que va a necesitar, y los estudiantes deben comprometerse en el tratamiento correcto de las interacciones en el aula, para hacer buen uso de los espacios, tiempos y recursos, teniendo claro el compromiso con el logro de los objetivos de aprendizaje. En este estudio se resalta la solicitud por espacios más amplios y otro tipo de mobiliario (los puestos individuales ya resultan incómodos). Un estudio reciente aborda la cuestión de cómo los espacios de aprendizaje son transformados, producidos y desarrollados (Kokko \& Hirsto, 2021)En cuanto a la presencia y empleo de distintos dispositivos tecnológicos en el aula, en general los profesores tienen sus propias posturas, no siempre desde perspectivas teóricas, sobre el uso de tecnologías en las clases. En una reciente investigación sobre el uso de dispositivos móviles en proceso de enseñanza aprendizaje en Instituciones universitarias (Valencia, Benjumea, Morales, Silva, 
y Betancur, 2018), revelan discrepancias en la aceptación de dispositivos móviles, pues algunos los ven como factores de distracción. Puede tratarse entonces de un problema abierto frente a la gestión de ambientes de aprendizaje, que seguramente deba abordarse desde los marcos teóricos actuales que involucran los contextos institucionales y las aproximaciones tecnológicas.

\section{Actividades de aprendizaje}

En los casos observados es muy marcado el apego de los profesores a la ruta de clase, y por ende a los textos guía, y el manejo de los tres momentos de clase (ya descritos), que se transforma muchas veces en afanes del profesor. Es muy probable que la dependencia de los textos guía sea responsable de la marcada tendencia de los profesores a estar dando explicaciones detalladas de los procesos algorítmicos y de las estrategias específicas para enfrentar los problemas planteados en los libros, desaprovechando oportunidades de plantear situaciones problema, que promuevan actividad matemática por parte de los estudiantes. Los afanes por cumplir con los tiempos marcados para cada momento de clase, genera dos

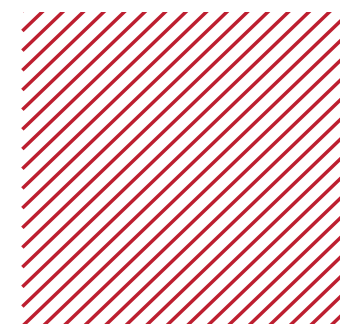
tipos de escenarios poco deseables, porque se interrumpe de manera algo abrupta el trabajo que se está haciendo, o se desaprovechan discusiones interesantes que se estén gestando antes del "cambio de momento". Según lo observado, es normal que el primer momento de la clase, destinado a revisar la preparación previa de la clase, se agote tratando de explicar las dudas de los estudiantes, y no se da la opción de un diagnóstico efectivo de conocimientos previos o sistemas de creencias, que podrían orientar mejor las decisiones a tomar en el desarrollo de la clase. El énfasis en los tratamientos procedimentales descuida un poco la oportunidad de promover procesos de pensamiento matemático a través de estrategias asociadas a la resolución de problemas.

Lo importante de una situación problema es que se pongan en juego los conocimientos previos del estudiante, se cuestionen y puedan modificarse, generando nuevos conocimientos. No es necesario que la situación tenga un contexto de la vida cotidiana, lo importante es que proponga un desafío a resolver. Los profesores suelen moverse entre dos extremos: trivializar las ideas matemáticas con el afán de llevarlas a contextos cotidianos, o acudir al formalismo y rigor con el cual los textos tradicionales plantean lo que sus autores etiquetan como problemas. Lo que realmente se ponen en juego con la idea de situación problema es la creatividad del profesor y su profunda claridad sobre los procesos de pensamiento que quiere activar. 
A nuestro juicio, de acuerdo con las observaciones, el nivel de compromiso de los estudiantes con su aprendizaje individual, en los tres grupos, es bajo en general; aquí seguramente hay materia para investigaciones contextualizadas, que se pregunten por las motivaciones de esta generación de nativos digitales, que parecen tener otros intereses y otras formas de aproximarse al conocimiento.

Finalmente queremos señalar, en esta categoría de análisis de las actividades de aprendizaje, algunos aspectos relevantes del proceso de evaluación. Tal como se programa y ejecuta en el curso, el énfasis recae en las calificaciones de pruebas y de tareas en general, que en mayor porcentaje se construyen por imitación del tipo de ejercicios que está propuesto en los textos. Además, no parece haber consenso en lo que se entiende por evaluación formativa. Los profesores incluyen bajo esta denominación la atención a cierto tipo de valores personales, como el comportamiento en clase o el cumplimiento con los deberes; la retroalimentación a los estudiantes se lleva a cabo en momentos muy puntuales, y casi siempre centrada en la ejecución de procedimientos. La investigación en educación plantea que la finalidad de la evaluación formativa es estrictamente pedagógica: regular el proceso de enseñanza- aprendizaje para adaptar o ajustar las condiciones pedagógicas (estrategias, actividades) en servicio del aprendizaje de los estudiantes. Una adecuada evaluación formativa requiere romper el ámbito de la evaluación cuantitativa como único recurso de retroalimentación a las acciones de los estudiantes; un ambiente de aprendizaje que tenga presente adecuados procesos de retroalimentación en la evaluación que le permitan al estudiante tomar conciencia del estado de sus conocimientos frente a los estándares del curso, elimina niveles de estrés y promueve mayores niveles de rendimiento académico (Castejón, Romero y López, 2015).

\section{Recursos para el aprendizaje}

La observación nos deja un llamado de atención respecto a la necesidad de revisar la propuesta de evaluación del programa del curso, pensando en una mayor coherencia con los objetivos y la metodología que se describen en el mismo. Nos parece claro que se necesitan decisiones institucionales, porque se percibe cierta reticencia de los profesores catedráticos a tomar iniciativas frente a aspectos trascendentales en el desarrollo de sus actividades de clase, como los son la evaluación y el manejo de los tiempos. Muchas veces la preocupación por cumplir con el programa, impidió el desarrollo de discusiones o de planteamientos alternativos que ofrecían, en apariencia, aportes significativos para el alcance de los objetivos de aprendizaje. Es claro que, aunque los problemas que surgen en el proceso de enseñanza aprendizaje son en su mayoría de orden didáctico, es imposible deslindarse de los contextos institucionales. 
Como ya hemos señalado, la investigación educativa en las últimas dos décadas le otorga un rol clave a las tecnologías en las construcciones conceptuales y teóricas en matemáticas. La facilidad que introducen los nuevos sistemas de cálculo para ejecutar eficientemente tareas rutinarias, plantea retos en los diseños curriculares y en el diseño de tareas, para centrarse en el desarrollo de procesos centrales de pensamiento y poner en juego la capacidad de razonamiento de los estudiantes. Inclusive, con preguntas adecuadas, es posible explorar de manera temprana conceptos que se consideraban pertenecientes a niveles superiores de escolaridad. Este hecho tiene repercusiones fundamentales en la programación de los contenidos de los cursos y el manejo de los tiempos para los mismos. Las observaciones nos muestran un acercamiento todavía muy limitado al empleo de recursos tecnológicos en los cursos de Álgebra y funciones; casi que todo el peso lo tiene el empleo de Moodle, que es más una herramienta para la logística de las actividades del profesor, pero se desaprovecha todo el potencial de los Sistemas de Geometría Dinámica (SGD), como GeoGebra. Los estudios mencionados en el marco teórico ya muestran resultados sobre la incidencia de los SGD en la amplificación del ambiente de aprendizaje y en la inmersión en los diversos sistemas de representación, lo cual influye directamente en el desarrollo de los procesos centrales de pensamiento matemático. Existen incluso reportes específicos sobre el empleo de GeoGebra (Santos-Trigo et al., 2019, Mudaly \& Fletcher, 2019; Olsson, 2019; Zulnaidi, Oktavika, \& Hidayat, 2020).

De acuerdo con nuestras observaciones, debemos señalar que es posible que nuestros profesores no le estén prestando la atención necesaria a las dificultades de los estudiantes con el manejo del lenguaje técnico del curso, con lo cual están descuidando también la dimensión semiótica de la actividad matemática, considerada en las investigaciones educativas como trascendental para la adquisición del conocimiento matemático. Son dos las tendencias que enmarcan esta desatención: i) la alta dependencia de los textos guía para el desarrollo del curso. Estos textos comerciales están escritos pensando en los profesores de matemáticas que van a transmitir unos conocimientos, ceñidos a la presentación formal de la matemática como ciencia deductiva, con todo el rigor del lenguaje y de la estructura axiomática-demostrativa; ii) la inclinación de los profesores por los discursos explicativos, que poco se apartan de la propuesta de los libros.

Lo que se puede notar en las observaciones es que los estudiantes recurren a estrategias nemotécnicas, para recordar recetas a las que no les pueden asignar sentido, pero que saben que les serán útiles en las evaluaciones "por imitación" que privilegian los profesores. Un

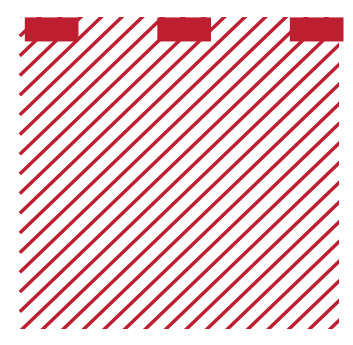


hecho importante de señalar de la revisión de los cuadernos, es que en ninguno de ellos se pudo evidenciar una anotación del estudiante que reflejara una reflexión propia o una duda importante; solo tenían registros idénticos de los enunciados de los textos o de los escritos del profesor en el tablero.

El concepto de función en particular ofrece un contexto ideal para poner en juego diversos sistemas de registros de representación semiótica y propiciar reflexiones interesantes sobre el lenguaje matemático.

Algo común que se observó, es que los tres profesores promueven la comunicación verbal de los estudiantes en la clase, propiciando espacios para que estos manifiesten por este medio sus ideas e inquietudes, lo cual hacen con mucha dificultad; es decir, a los problemas de los estudiantes con el lenguaje matemático se les pueden añadir sus problemas con el lenguaje natural.

\section{Conclusiones y recomendaciones}

La replicabilidad de un estudio como el que presentamos aquí, con la intención de buscar soluciones a problemas de aprendizaje, requiere tener presente lo que parece ser un imperativo en las condiciones actuales de la investigación educativa, y es la necesidad de centrarse en las prácticas institucionales que condicionan contenidos, normas y valores, tanto o más que atender al estudiante y su desarrollo cognitivo. Los ambientes de aprendizaje deben tener en cuenta características sociales y culturales de los actores de la interacción, por lo cual identificar en los estudiantes sus particularidades generacionales es importante. Los profesores nos vemos abocados entonces a enfrentar varios retos: $i$ ) incorporar un discurso tecnológico, articulado con los saberes matemáticos y prepararnos para un "diálogo inteligente con las herramientas" (Artigue, 2016); ii) experimentar nosotros mismos los potenciales y posibles falencias de las herramientas digitales (Santos-Trigo, 2019); iii) aprender a diseñar tareas digitales que extiendan y amplifiquen las características pedagógicas de los ambientes sin tecnología y analizar los cambios en contenidos matemáticos promovidos por tareas mediadas con tecnologías (Santos-Trigo, 2019).

Las universidades tienen que empezar a pensar en la renovación de sus espacios (tamaño de los salones, mesas de trabajo, equipos de cómputo, entre otros) y ofrecer programas de desarrollo profesional docente, que preparen a sus profesores para asumir los retos de la era digital y los involucre también con los resultados y tendencias en la investigación en educación.

Artigue (2016) señala en su conferencia que, a nivel universitario, la investigación en educación matemática reconstruye, en sus principios, los resultados de las teorías constructivistas y posibilita su incorporación en nuevos paradigmas. 
La era digital trae consigo otras propuestas en teorías de aprendizaje, como el denominado Conectivismo (Gutiérrez, 2012; Rønningsbakk, 2019), que trae planteamientos a considerar en un posible programa de actualización docente: i) las formas tradicionales de aprendizaje no le siguen el paso a la velocidad con que los cambios se producen; el aprendizaje es continuo y por ello el diseño de tecnologías de aprendizaje (sistemas en continua evolución) reemplazará el diseño instruccional; y el aprendizaje es complejo, ocurre en diferentes escenarios y los ambientes tradicionales son limitados en este sentido, pues no se desarrollan más allá del aula de clase; ii) hay cambios dramáticos en estrategias de aprendizaje y en las instituciones educativas; éstas últimas están ingresando en la modalidad de vender conocimiento y servicios de formación como productos que compiten en el mercado; los estudiantes empiezan a ser considerados como consumidores en lugar de aprendices.

En el contexto de nuestro estudio debemos señalar que nuestras observaciones, y las evaluaciones que los estudiantes hacen de los profesores, nos hablan muy bien de la idoneidad disciplinar y los valores humanos de P1, P2 y P3. Cabe además, resaltar los aspectos siguientes: i) la solicitud insistente de los profesores en cambiar los salones destinados al curso AyF; ii) la inclinación de los profesores a responder las inquietudes de los estudiantes recurriendo a explicaciones expositivas, dando poco lugar a otras estrategias que promuevan más la actividad del estudiante (investigación, discusión entre pares, formulación y validación de conjeturas, entre otras); iii) la tendencia a mediar las clases con el empleo del lenguaje riguroso de los textos guía, sin atender a la escasa comprensión que de ese lenguaje manifiestan los estudiantes (promoviendo así la memorización de rutinas procedimentales, a las que no se les asigna sentido); iv) la constante preocupación de los profesores por el manejo de los tiempos, debida a la presión que sienten por el cubrimiento del programa del curso (tendencia muy marcada en profesores contratistas). Se nota que esta misma presión afecta la reflexividad del profesor, porque la mayoría de las decisiones que toma frente a diferentes circunstancias de clase, están primordialmente influenciadas por su compromiso con el cronograma del día.

Uno de los aspectos observados y destacados en nuestras discusiones, es el de las relaciones entre el lenguaje natural y el lenguaje matemático. Seguramente hay que profundizar en el papel que entra a jugar el nuevo lenguaje impuesto por las tecnologías digitales. 


\section{Colofón}

La metodología de este estudio facilitó la socialización de los hallazgos y la discusión de propuestas de nuevos enfoques para el curso, con el respaldo de un marco teórico enriquecido en la práctica. Estos resultados se aunaron a otras iniciativas institucionales que produjeron reformas significativas. El grupo de profesores del curso de Álgebra y funciones se ha venido constituyendo en una comunidad de práctica, en constante actividad académica y trabajo colaborativo. Se ha avanzado considerablemente en el diseño de mecanismos e instrumentos de evaluación desde la perspectiva de competencias y se inició un proceso de apropiación y empleo de recursos digitales, que ha sido potencializado por la necesidad del trabajo remoto durante la pandemia. Estamos pendientes de poder analizar los datos que arrojen estas reformas sobre el impacto en la solución del problema de bajo rendimiento académico.

\section{Referencias bibliográficas}

Aguilar-Magallón, D. (2018). La Formulación y Resolución de Problemas Matemáticos en un Ambiente que Promueve el Uso de Tecnologías Digitales. CINVESTAV.

Artigue, M. (2011). Tecnología y enseñanza de las matemáticas: desarrollo y aportes de la aproximación instrumental. Cuadernos de Investigación y Formación En Educación Matemática, 6(8), 13-33.

Artigue, M. (2016). Mathematics Education Research at University Level: Achievements and Challenges. First Conference of International Network for Didactic Research in University Mathematics.

Artigue, M. (2017). The Challenging Relationship Between Fundamental Research and Action in Mathematics Education. In G. Kaiser (Ed.), Proceedings of the 13th International Congress on Mathematical Education (pp. 145-163). Springer International Publishing.

Bosch, M., Fonseca, C., y Gascón, J. (2004). Incompletitud de las organizaciones matemáticas locales en las instituciones escolares. Recherche En Didactique de Matematiques, 24, 205-250.

Castejón, F., Romero, R., y López, V. (2015). Divergencias del alumnado y del profesorado universitario sobre las dificultades para aplicar la evaluación formativa. RELIEVE - Revista Electrónica de Investigación y Evaluación Educativa, 21(1), 1-17.

D’amore, B. (2004). Conceptualización, registros de representaciones semióticas y noética: interacciones constructivistas en el aprendizaje de los conceptos matemáticos e hipótesis sobre algunos factores que inhiben la devolución. Uno. Revista Didáctica de Las Matemáticas, 35, 90-106. 
Dominguez, G. L., \& Cerqueira, J. (2021). A Theoretical Model of Mathematics for Teaching the Concept of Function. The Mathematics Enthusiast, 18(3), 535-577. https://scholarworks.umt.edu/tme/vol18/iss3/8

Drijvers, P., Kieran, C., Mariotti, M.-A., Ainley, J., Andresen, M., Chan, Y. C., Dana-Picard, T., Gueudet, G., Kidron, I., Leung, A., \& Meagher, M. (2009). Integrating Technology into Mathematics Education: Theoretical Perspectives. In C. Hoyles \& J.-B. Lagrange (Eds.), Mathematics Education and Technology-Rethinking the Terrain:The 17thICMIStudy (pp. 89-132). Springer US. https://doi.org/10.1007/978-1-4419-0146-0_7

Gueudet, G., Bosch, M., DiSessa, A. A., Kwon, O. N., \& Verschaffel, L. (2016). Transitions in Mathematics Education. Springer International Publishing. https://doi.org/10.1007/978-3-319-31622-2

Gutiérrez, L. (2012). Conectivismo como teoría de aprendizaje: conceptos, ideas, y posibles limitaciones. Revista Educación y Tecnología, 1, 111-122.

Hegedus, S. J., \& Moreno-Armella, L. (2011). The emergence of mathematical structures. Educational Studies in Mathematics, 77(2), 369-388. https:// doi.org/10.1007/s10649-010-9297-7

Hitt, F., \& González-Martín, A. S. (2016). Generalization, Covariation, Functions, and Calculus. In Á. Gutiérrez, G. C. Leder, \& P. Boero (Eds.), The Second Handbook of Research on the Psychology of Mathematics Education: The Journey Continues (pp. 3-38). SensePublishers. https://doi. org/10.1007/978-94-6300-561-6_1

Kokko, A. K., \& Hirsto, L. (2021). From physical spaces to learning environments: processes in which physical spaces are transformed into learning environments. Learning Environments Research, 24(1), 71-85. https://doi.org/10.1007/s10984-020-09315-0

MEN. (2014). Foro educativo nacional. Ciudadanos matemáticamente competentes. Magisterio. http://www.iecov.edu.co/documentos/documento_orientador_foro_educativo_2014.pdf

Moreno-Armella, L., \& Brady, C. (2018). Technological Supports for Mathematical Thinking and Learning: Co-action and Designing to Democratize Access to Powerful Ideas. In L. Ball, P. Drijvers, S. Ladel, H.-S. Siller, M. Tabach, \& C. Vale (Eds.), Uses of Technology in Primary and Secondary Mathematics Education: Tools, Topics and Trends (pp. 339-350). Springer International Publishing. https://doi. org/10.1007/978-3-319-76575-4_19

Mudaly, V., \& Fletcher, T. (2019). The effectiveness of geogebra when teaching linear functions using the Ipad. Problems of Education in the 21st Century, 77(1), 55-81. https://doi.org/10.33225/pec/19.77.55

Nyikahadzoyi, M. R. (2015). Teachers' knowledge of the concept of a function: a theoretical framework. International Journal of Science and Mathematics Education, 13(S2), 261-283. https://doi.org/10.1007/ $\underline{\text { s10763-013-9486-9 }}$ 
Olsson, J. (2019). Relations Between Task Design and Students' Utilization of GeoGebra. Digital Experiences in Mathematics Education. https://doi. org/10.1007/s40751-019-00051-6

Rach, S., \& Heinze, A. (2017). The Transition from School to University in Mathematics: Which Influence Do School-Related Variables Have? International Journal of Science and Mathematics Education, 15(7), 1343-1363. https://doi.org/10.1007/s10763-016-9744-8

Rodríguez-Cisneros, L. M., y Perdomo-Díaz, J. (2019). Transición del bachillerato a universidad en matemáticas desde la visión del docente. $X V$ Conferencia Interamericana de Educación Matemática CIAEM, 5-10 de Mayo, Medellin, Colombia., 1-8.

Rønningsbakk, L. (2019). Knowing What, Knowing How, or Knowing Where? How Technology Challenges Concepts of Knowledge (pp. 616-624). https:// doi.org/10.1007/978-3-030-35343-8_65

Santos-Trigo, M. (2019). Mathematical Problem Solving and the Use of Digital Technologies. In P. Liljedahl \& M. Santos-Trigo (Eds.), Mathematical Problem Solving: Current Themes, Trends, and Research (pp. 63-89). Springer International Publishing. https://doi. org/10.1007/978-3-030-10472-6_4

Santos-Trigo, M., Aguilar-Magallón, D., \& Reyes-Martínez, I. (2019). A Mathematical Problem-Solving Approach Based on Digital Technology Affordances to Represent, Explore, and Solve problems via Geometric Reasoning. In P. Felmer, P. Liljedahl, \& B. Koichu (Eds.), Problem Solving in Mathematics Instruction and Teacher Professional Development (pp. 145-166). Springer International Publishing. https:// doi.org/10.1007/978-3-030-29215-7_8

Santos-Trigo, M., \& Moreno-Armella, L. (2016). The Use of Digital Technology to Frame and Foster Learners' Problem-Solving Experiences. In P. Felmer, E. Pehkonen, \& J. Kilpatrick (Eds.), Posing and Solving Mathematical Problems: Advances and New Perspectives (pp. 189-207). Springer International Publishing. https://doi. org/10.1007/978-3-319-28023-3_12

Santos-Trigo, M., Reyes-Martínez, I., \& Aguilar-Magallón, D. (2016). Digital Technologies and a Modeling Approach to Learn Mathematics and Develop Problem Solving Competencies. In L. Uden, D. Liberona, \& B. Feldmann (Eds.), Learning Technology for Education in Cloud -The Changing Face of Education (pp. 193-206). Springer International Publishing.

Santos-Trigo, M., y Benítez Mojica, D. (2003). Herramientas tecnológicas en el desarrollo de sistemas de representación para la resolución de problemas. Perfiles Educativos [En Linea], XXV(100), 23-41. https://www. redalyc.org/articulo.oa?id=13210003 
Sierpinska, A. (1992). The concept of function: some aspects of epistemology and pedagogy. MAA Notes, 25, 25-58.

Tall, D. (1992). The transition to Advanced Mathematical Thinking: Functions, Limits, Infinity, and proof. In Macmillan (Ed.), Handbook of Research on Mathematics Teaching and Learning.

Tall, D. (2000). Cognitive development in advanced mathematics using technology. Mathematics Education Research Journal, 12(3), 196-218. https://doi.org/10.1007/BF03217085

Valencia, A., Benjumea, M., Morales, D., Silva, A., y Betancur, P. (2018). Actitudes de docentes universitarios frente al uso de dispositivos móviles con fines académicos. Revista Mexicana de Investigación Educativa, 23(78), 761-790.

Zulnaidi, H., Oktavika, E., \& Hidayat, R. (2020). Effect of use of GeoGebra on achievement of high school mathematics students. Education and Information Technologies, 25(1), 51-72. https://doi.org/10.1007/ s10639-019-09899-y

\section{Notas}

${ }^{1} \mathrm{Mg}$. en Sociedad de la información y el conocimiento, Universidad Oberta de Cataluña, Barcelona, España. Profesor Departamento de Pedagogía, Universidad Icesi, Cali, Colombia. Correo electrónico: hataquez@ icesi.edu.co ORCID: 0000-0003-3298-9979

${ }^{2}$ Mg. en matemáticas, Universidad del Valle, Cali, Colombia. Profesor

Departamento de matemáticas y estadística, Universidad Icesi, Cali, Colombia. Correo electrónico: hyaker@icesi.edu.co ORCID: 0000-0002-8766-4410

${ }^{3}$ Doctora en Dirección de Empresas, Universidad de Valencia, Valencia, España. Profesora Departamento de gestión organizacional, Universidad Icesi, Cali, Colombia. Correo electrónico: angela.bedoya@u. icesi.edu.co ORCID: 0000-0002-5027-2560

${ }^{4}$ Mg. en Educación, Universidad Icesi, Cali, Colombia. Profesor, Institución educativa José Antonio Galán, Yumbo, Valle. Correo electrónico: profealexparra@gmail.com ORCID: 0000-0002-3258-4165 